\title{
9 Verwalten von Notizen und Fundstücken
}

\subsection{Digitale Zettelkästen}

\subsubsection{Webbasierte Zettelkästen}

\section{Evernote}

Evernote ist so etwas wie Ihr digitaler Schuhkarton, für alles was vielleicht noch nützlich sein kann. All das, was man zu Hause in die Schüssel auf der Flurkommode wirft, wie z. B. Zettel, Flyer, Konzertdaten, Notizen (zum leckeren Wein von heute Abend) etc. können Sie auch mit Evernote sammeln - und wiederfinden! Sie können Notizen eingeben, Webadressen speichern, Webschnipsel (Text, Bilder oder Videos) sammeln, Sprachnotizen oder Photos speichern. Alle diese Inhalte können Sie in Notizbücher sortieren oder einfach mit Tags versehen. Für mich das beste Feature bei Evernote: die OCR-Erfassung (Optical Character Recognition) von Bildern. Mit Hilfe dieser Technik kann Evernote alle Texte auf Ihren Bilder als Text auslesen und durchsuchbar machen.

Sie gehen durch die Stadt und sehen ein Veranstaltungsplakat und denken, „Ja, da will ich gern hin“. Machen Sie einfach ein Photo von dem Plakat, und Evernote wird den Text auf dem Plakat für Sie im Volltext durchsuchbar machen. Das ist auch sehr nützlich für Visitenkarten. Aber auch handgeschriebene Notizen können, so die Schrift es zulässt, von Evernote ausgelesen werden.

Evernote gibt es als Webdienst, den Sie über Ihren Browser aufrufen und über ein Add-on einbinden können, als Desktop-Client, der sich mit dem Webdienst synchronisiert und Ihnen Back-up und Offlinenutzung bietet und als App für das Smartphone oder den Tablet-PC. Gerade die zusätzliche Nutzung über das Smartphone mit seiner Foto-Funktion ist oft eine schnelle und handliche Lösung, neue Inhalte in Evernote „zu werfen“.

Evernote bietet einen kostenlosen Account und einen kostenpflichtigen Premium-Account an. Der Premium-Account beinhaltet mehr monatlichen Speicherplatz, einige zusätzliche Funktionen und die Offline-Nutzung von Notizbüchern auf mobilen Endgeräten. Die hier beschriebenen Funktionen beziehen sich auf den kostenlosen Account von Evernote.

Der kostenlose Account beschränkt das monatliche Speicher- bzw. Upload-Volumen. Das Upload-Volumen ist begrenzt auf 60 MB und beinhaltet alle Synchronisierungsprozesse mit der Webplattform. Nutzt man Evernote viel und gern auch mit Bilddateien oder anderen größeren Dateiformaten, ist dies Volumen leicht mal vor Monatsende erschöpft. Auch die Offline-Nutzung ist beim kostenlosen Account ausschließlich über den Desktop-Client möglich, nicht aber über die App.

\section{Neue Inhalte aufnehmen}

Browser/Evernote-Web

Um über den Browser neue Inhalte in Evernote einzugeben, können Sie entweder die Notizfunktionen der Webseite (Evernote-Web) nutzen oder das Add-on (Evernote Clipper), mit dem Sie Ihren Browser erweitern können. Wenn Sie Bilder von einer Webseite übernehmen wollen, können Sie diese auch einfach per drag \& drop in Evernote ,ziehen“.

\section{Lernziele}

In diesem Kapitel lernen Sie, Textstellen, Bilder und Videos schnell abzulegen, zu erschließen und wieder zu finden.

Inhalt dieses Kapitels:

- Digitale Zettelkästen im Web

- Digitale Zettelkästen auf dem Desktop

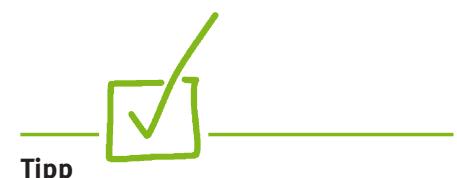
Anwendungsbeispiele für das Einbinden von Evernote in verschiedene Workflows können Sie auf diesen beiden Seiten finden: Offizieller Blog von Evernote: [evernote-de.tumblr.com] Blog „Evernote für Pfiffige“: [evernote-tips.posterous.com] 
Die Webseite bietet die Möglichkeit, formatierte Notizen zu erstellen (1), die vorhandenen Inhalte zu verwalten (2), zu durchsuchen (3) und zu bearbeiten.

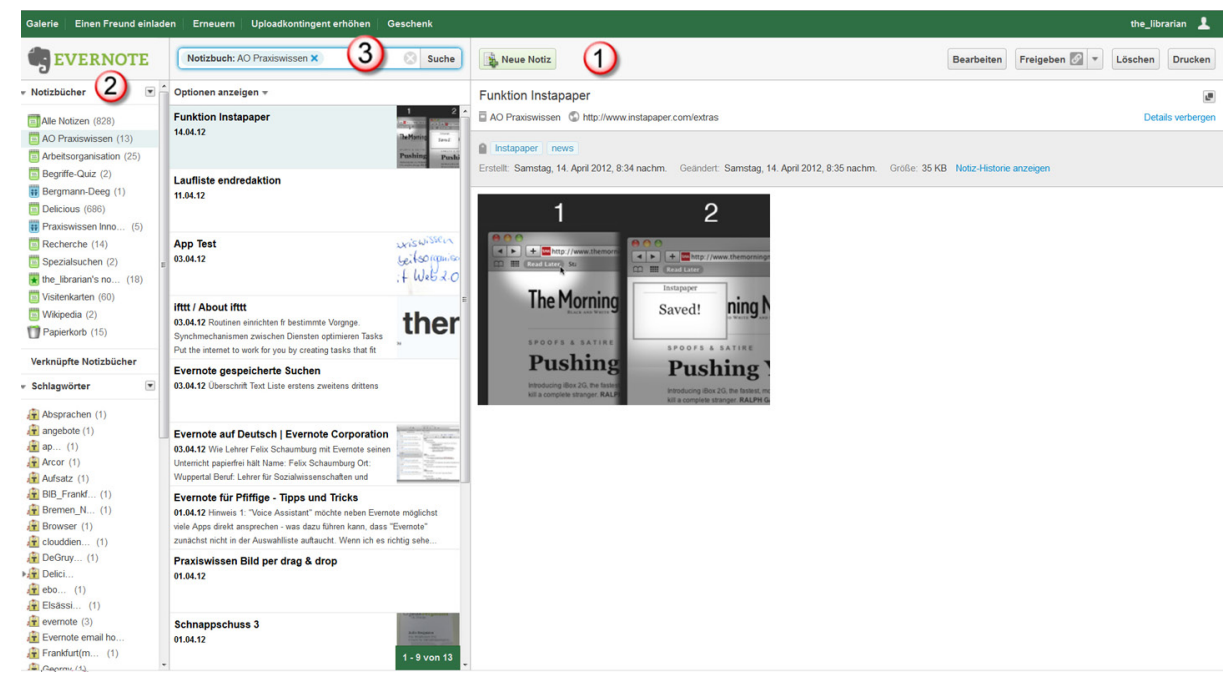

Um ein Bild von einer Webseite per drag \& drop in Evernote zu übernehmen, fasse ich das Bild mit der Maus an und ziehe es in die Notiz im Reiter „Evernote“.

Mit Hilfe des Add-on können Sie markierte Textstellen oder Bilder sowie die Webseite als Lesezeichen mit einem Klick in Evernote speichern. Es erscheint ein Popup, in dem Sie weitere Informationen eingeben können, wie den Speicherort (Notizbuch), Tags, eigene zusätzliche Notizen etc.

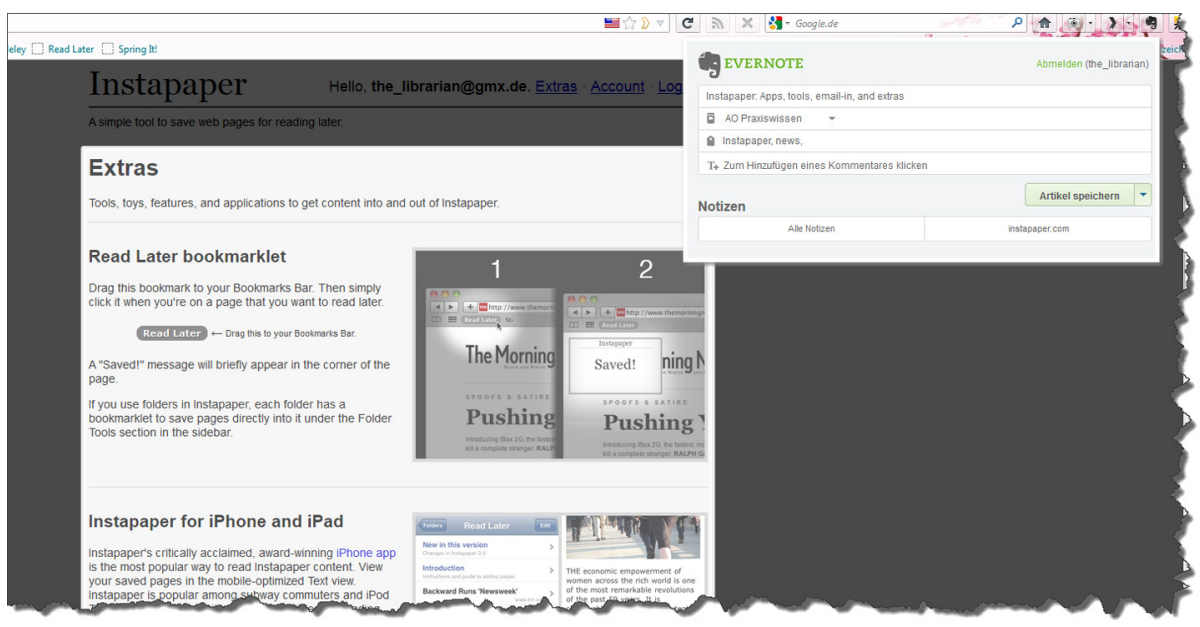

\section{Einstellungen des Add-on anpassen}

In den Einstellungen des Add-on können Sie definieren, wo ihr Inhalt gespeichert werden soll: in den Desktop-Client oder in den Webdienst, in welchen Ordner u.a. Um die Einstellungen des Add-on zu bearbeiten, gehen Sie in die Übersicht der Erweiterungen bzw. Add-ons in Ihrem Browser, und wenn Sie Evernote auswählen, erscheint ein Button „Einstellungen“. Klicken Sie diesen an, können Sie die Einstellungen verändern. 
Desktop

Mit dem Desktop-Client können Sie verschiedene Arten von neuen Notizen erstellen:

- getippte Notizen

- handschriftliche Notizen

- Webcam-Notizen

- Audio-Notizen

Wie in allen Oberflächen von Evernote, lassen sich in jeder Notiz natürlich Elemente verbinden, verlinken oder kombinieren. So können Sie Ihrer Notiz z. B. ein Bild oder einen Weblink hinzufügen.

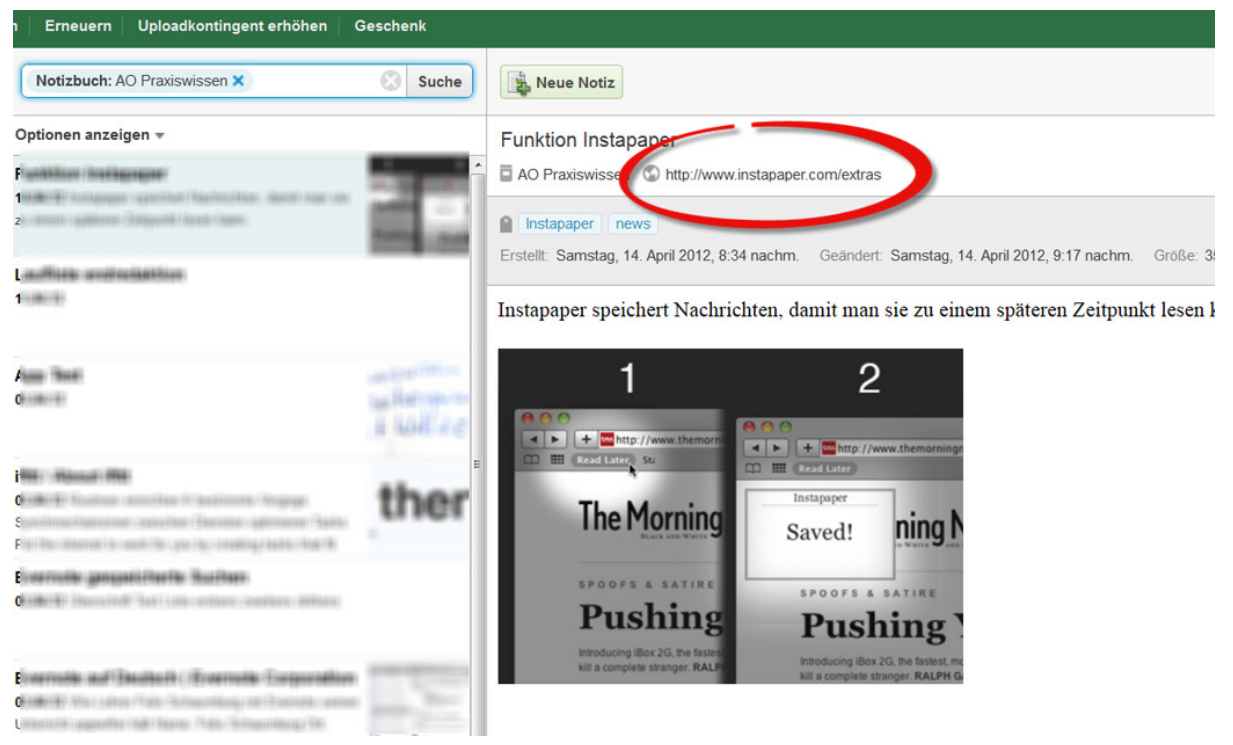

App

Evernote-Apps gibt es für Android, Apple (IOS), Blackberry und Windows Phone 8. Um Inhalte über die App hinzuzufügen, können Sie folgende Funktionen nutzen:

\section{Sprachnotizen}

Durch Spracherkennung können Sie so auch Notizen „diktieren“. Die gesprochenen Inhalte werden in Text umgewandelt. Ob diese Funktion verfügbar ist, hängt von Ihrem Endgerät ab. In der App wird sichtbar gemacht, wenn es beide Sprachoptionen für Ihr Gerät gibt.

\section{Schnappschuss}

Mit der Schnappschuss-Funktion können Sie Fotos von Plakaten, Notizen, Visitenkarten, Tafelanschriften, Flipchartblättern, Weinetiketten etc. machen. Sobald Ihr Bild Text enthält, wird es nicht nur als Bild gespeichert, sondern durch OCR-Erkennung auch durchsuchbar gemacht. Dies funktioniert sehr gut bei gedruckten Texten, geht aber auch bei handschriftlichen Notizen. Diese müssen aber entsprechend gut „lesbar" sein, damit die Erkennung funktioniert.

Ab dem 25. September 2012 bietet die Firma Moleskine in Zusammenarbeit mit Evernote spezielle Notizbücher an, die das Abfotografieren und „Erkennen“ handschriftlicher Notizen, durch gesetzte Rasterpunkte im Papier des Notizbuches, in der Qualität verbessern sollen.

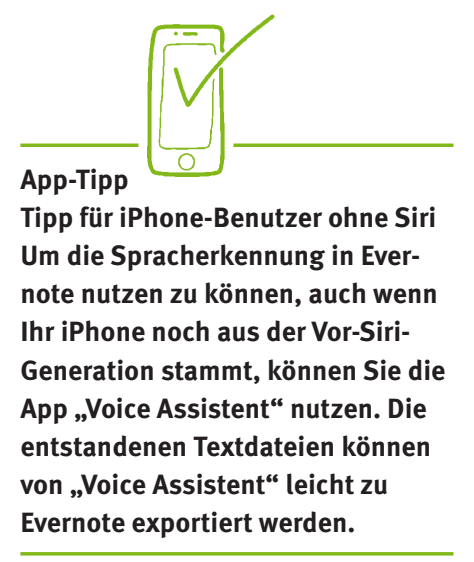


Bild aus Bildfunktion (Alben) des Smartphones übernehmen

Sie können auch ein auf Ihrem Gerät bereits vorhandenes Foto als Evernote-Notiz speichern. Dieses wird genau wie alle anderen Bilddateien in Evernote durch OCRErkennung durchsuchbar gemacht.

Getippte Notizen

Natürlich können Sie hier ganz klassisch eine Notiz eintippen.

Ortserkennung

Bei Notizen, die Sie mit Hilfe der App machen, wird zusätzlich zu Datum und Zeit auch der Standort gespeichert. Bei den automatischen Vorschlägen zum Notiztitel, die Evernote bei der Eingabe einer neuen Notiz anbietet, werden diese Daten hinzugezogen, um auch bei schnell abgespeicherten Notizen einen individuellen Titel zu generieren. Ebenfalls hinzugezogen werden Einträge aus Ihrem Kalender. Steht dort z. B. ein Termineintrag für die Konferenz, auf der Sie gerade sind, würde Evernote bei den Notiztitel-Vorschlägen z. B. vorschlagen: Notiz zu Konferenz XY in Berlin.

In den Notiz-Details würden dann das genaue Erfassungsdatum, Zeit und Ort gespeichert bzw. suchbar sein.

OCR-Verarbeitung

Evernote benötigt ein wenig Zeit, um neue Bild-Notizen mit Hilfe von OCR durchsuchbar zu machen. Bei meinen Tests mit der kostenlosen Version von Evernote benötigte Evernote 30-60 Minuten für die Bearbeitung.

Evernote verspricht Premiumkunden eine schnellere Bearbeitungszeit für die OCR-Erkennung.

Notizen aus anderen Apps übergeben

Evernote kann von vielen Apps als Export-Ort angesprochen werden. So können Sie z. B. eine Skizze zeichnen oder eine Notiz in einer anderen App verfassen und Sie dann an Evernote senden. Dadurch sind noch viele andere Formen der Eingabe von Notizen möglich.

Notiz per E-Mail senden

Sie können auch eine Notiz per E-Mail senden. Dabei können Sie Anhänge mitsenden. Durch die Betreffzeile ihrer Mail können Sie definieren, welchen Titel Ihre Notiz haben soll, in welches Notizbuch sie abgespeichert werden soll und mit welchen Tags Sie die Notiz versehen möchten.

Beispiel: Agenda Baumeeting @Bauplanung \#inneneinrichtung \#trockenbau

Dabei markiert das @ ein vorhandenes Notizbuch und \# kennzeichnet die Tags.

Die E-Mailadresse, an die Sie Ihre Inhalte senden können, finden Sie in Ihren Kontoeinstellungen.

Inhalte verwalten und wiederfinden

Evernote-Web

Die Webseite von Evernote bietet Ihnen die Möglichkeit, neue Notizbücher zu erstellen, vorhandene Inhalte und Notizbücher zu bearbeiten und nach Inhalten zu suchen. Auch die Freigabe von Notizbüchern können Sie hier verwalten. 
Die Darstellung ist in drei Spalten aufgeteilt. Links finden Sie die Notizbücher und Tags (1), in der Mitte die Liste (2) der einzelnen Notizen (Anzeige je nach Auswahl links bzw. Suche), rechts befindet sich die Detailansicht für Notizen (3). Dies ist gleichzeitig das Bearbeitungsfenster für Notizen.

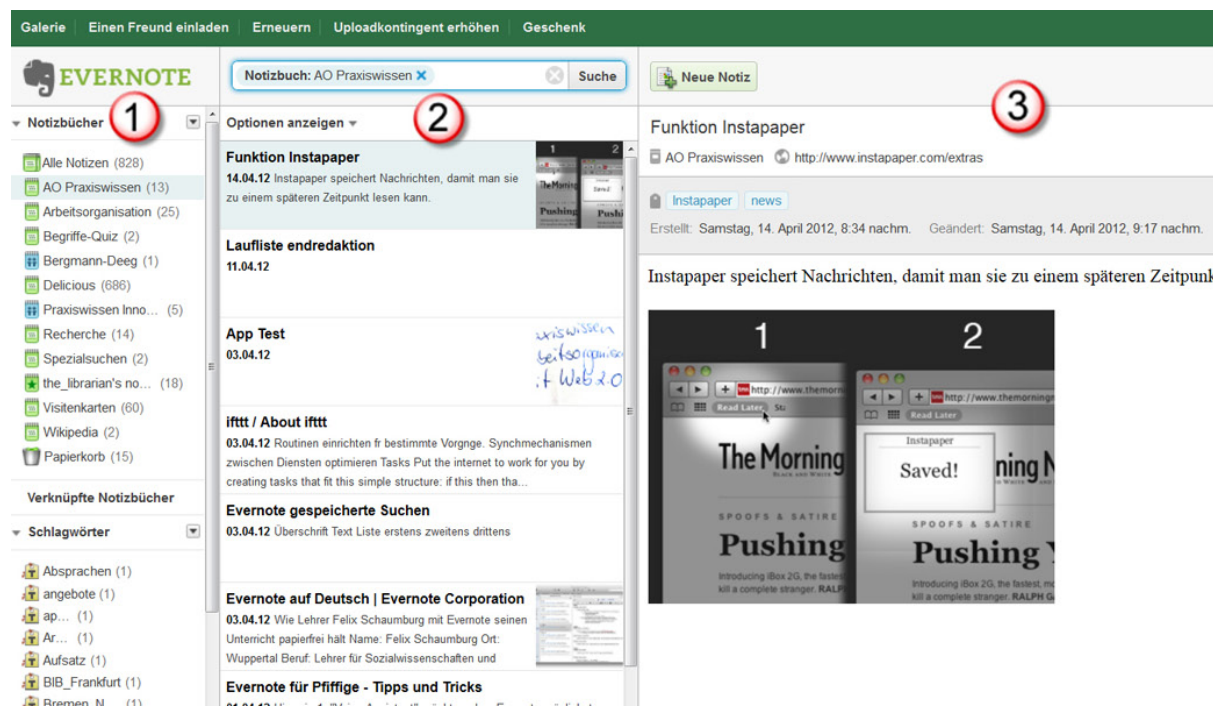

In der linken Spalte können Sie Ihre Notizbücher sortieren, durch Anklicken öffnen, neue Notizbücher erstellen, Notizbücher zu Stapeln versammeln oder Notizbücher freigeben. Das Kontextmenü erhalten Sie beim Klicken auf den kleinen Pfeil hinter dem Namen des Notizbuches.

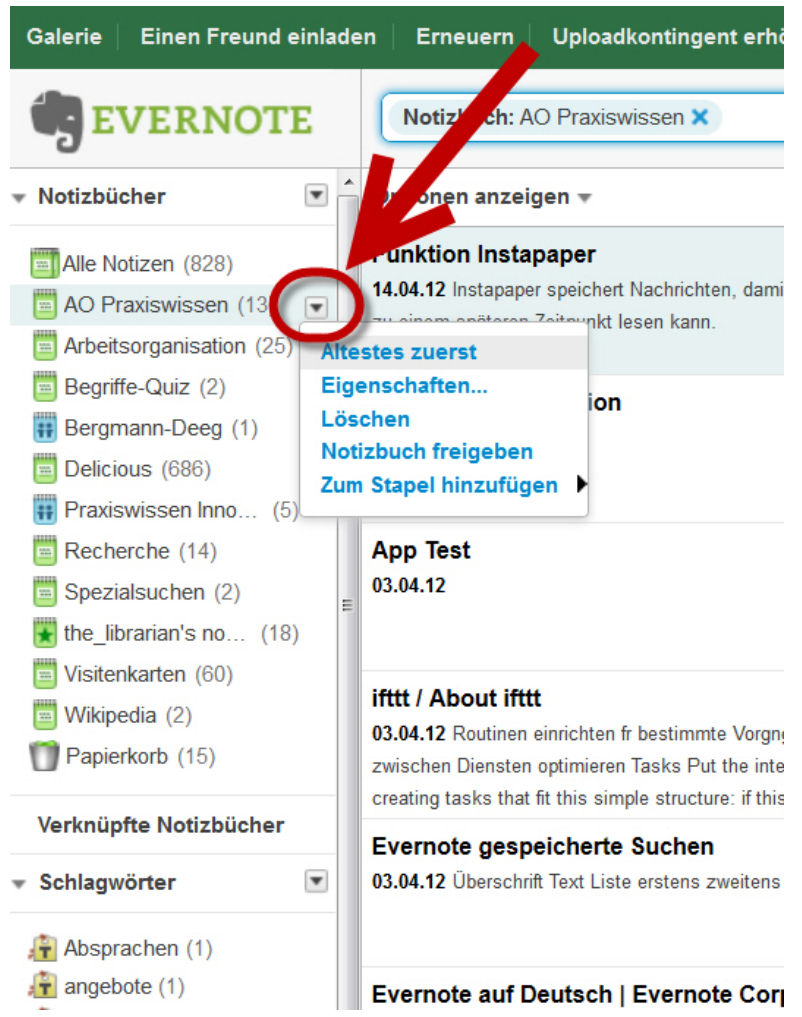


In der mittleren Spalte sind die einzelnen Notizen als Liste dargestellt. Die dargestellten Notizen werden entsprechend Ihrer Auswahl angezeigt. Klicken Sie auf ein Notizbuch, werden die Inhalte des Notizbuches dargestellt, wählen Sie „alle Notizen anzeigen", werden alle in chronologischer Reihenfolge gezeigt, führen Sie eine Suche durch, werden hier die Ergebnisse angezeigt. Die Sortierung der Liste kann über das Menü „Optionen anzeigen“ verändert werden.

Um einzelne Notizen in einem Notizbuch abzulegen, kann man die Notiz einfach per drag \& drop auf das Notizbuch ziehen.

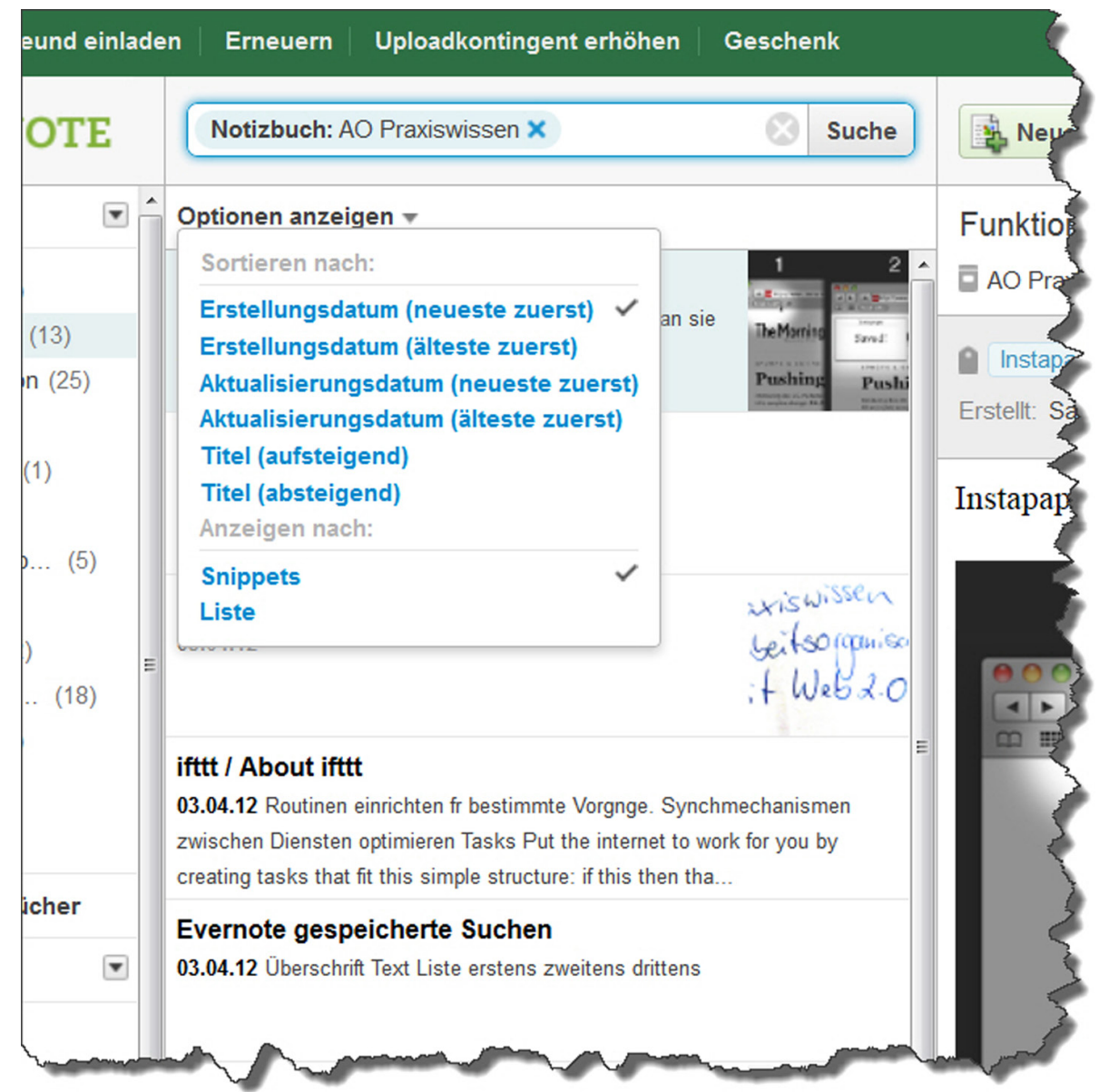


Die rechte Spalte ist für die Einzelbetrachtung der Notizen vorgesehen und gibt Ihnen die Möglichkeit, die Notiz zu bearbeiten (1), Bilder (2), Verlinkungen (3) und Tags (4) hinzuzufügen oder den Notizverlauf (5) anzuzeigen. Das Anzeigen des Notizverlaufes ist ein Premium-Feature, das nur angezeigt wird, wenn Sie einen kostenpflichtigen Premium-Account besitzen.

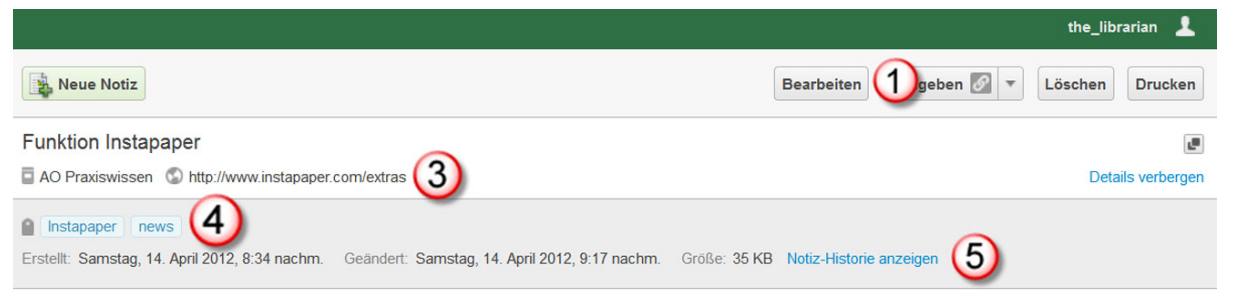

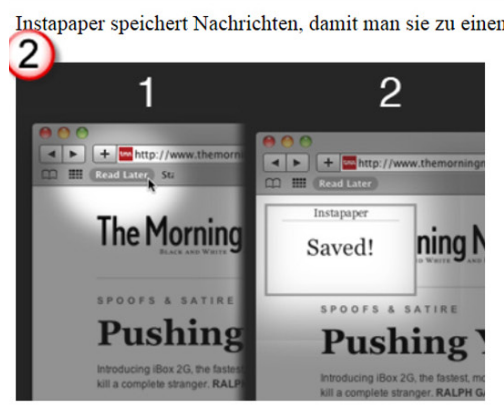

Die Bearbeitungsfunktion unterstützt das Formatieren des Textes mit den üblichen Office-Grundfunktionen, wie Schriftgröße, Schriftart und -farbe etc. Zusätzlich ist es möglich, Checkboxen einzufügen, um z. B. ToDo-Listen zu erstellen.

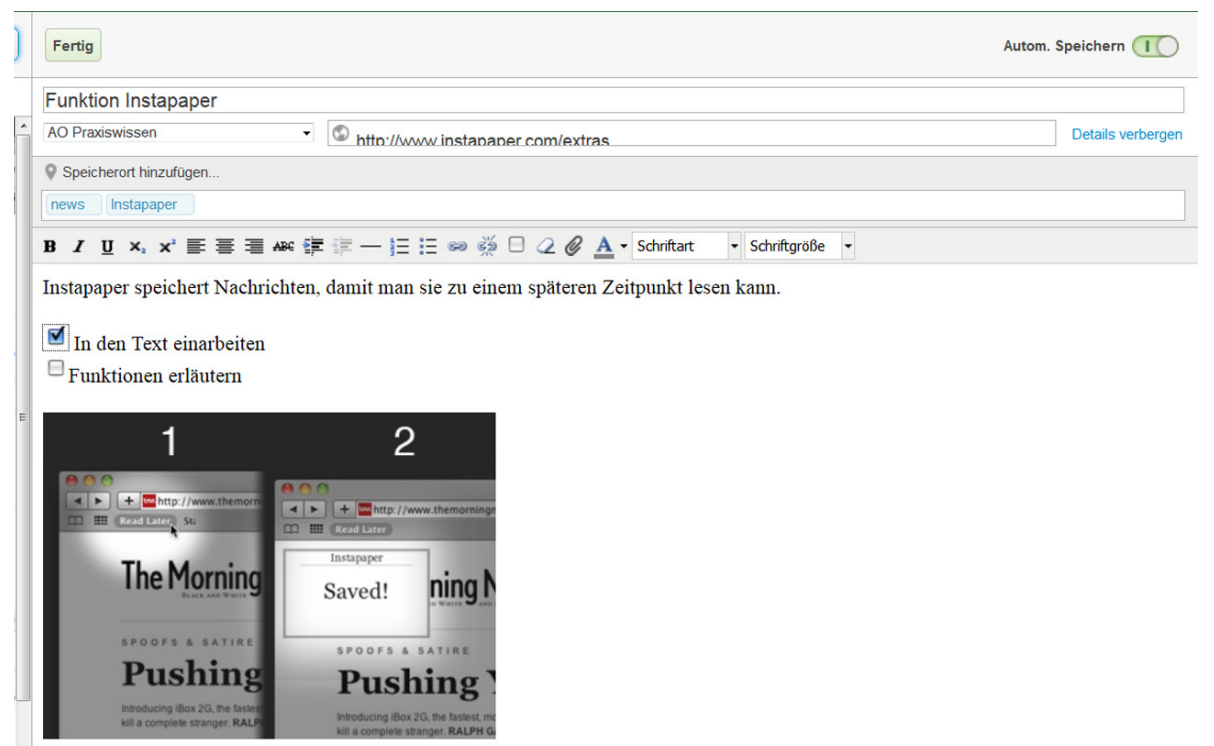


Desktop

Die Oberfläche des Desktop-Client ist weitestgehend genauso aufgeteilt wie EvernoteWeb. Linke Spalte für Notizbücher, mittlere Spalte für die Listenansicht der Notizen, rechte Spalte für die Einzelansicht und Bearbeitung.

Darüber hinaus verfügt die Desktop-Version von Evernote noch über einige weitere Funktionen.

Zunächst gibt es einen extra Button, um den Desktop-Client mit Evernote-Web zu synchronisieren. Evernote-Web ist der zentrale Speicherort, mit dem sich alle Ihre Endgeräte, auf denen Sie Evernote verwenden, synchronisieren. Dies können Sie entweder manuell anstoßen oder in den Einstellungen unter Funktionen $\rightarrow$ Optionen automatisieren.

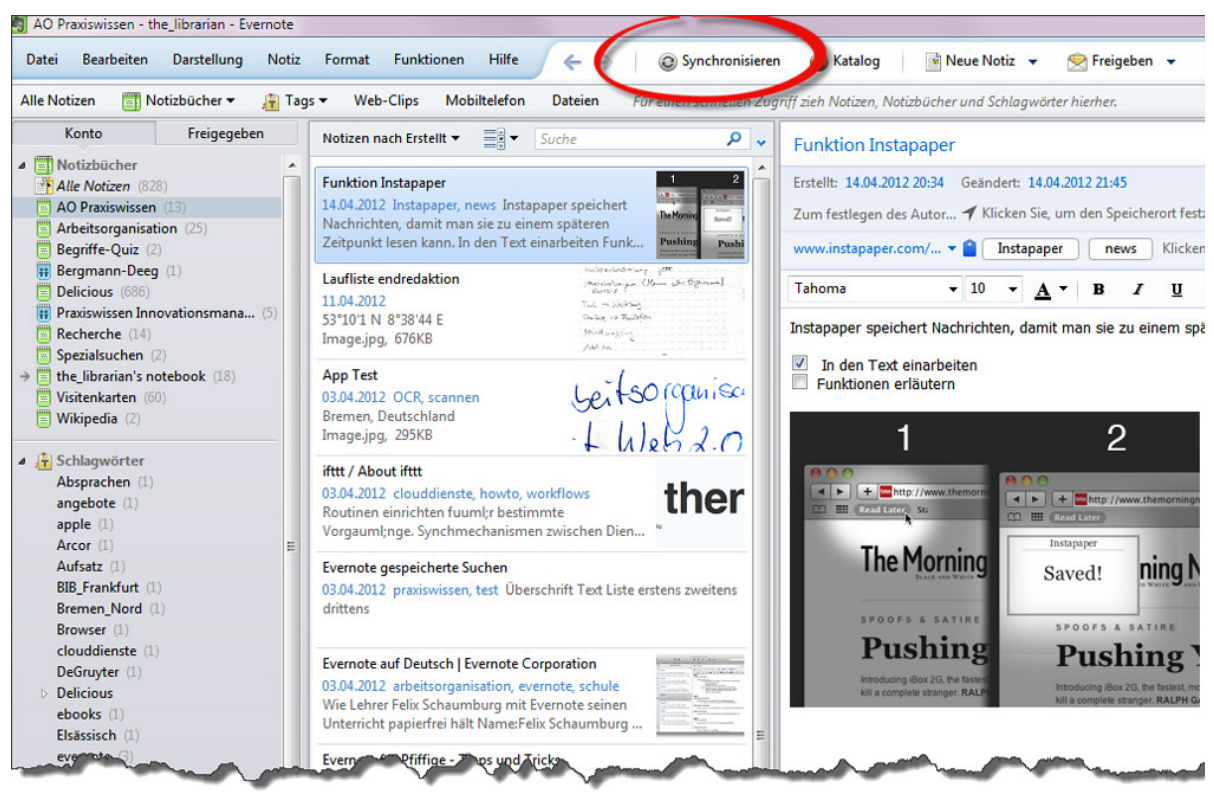

Beim Erstellen neuer Notizbücher unterscheidet der Desktop-Client zwischen lokalen und synchronisierten Notizbüchern. Sie können also auch Inhalte speichern, ohne sie mit Evernote-Web zu synchronisieren.

Grundsätzlich ist das Haupt-Menü in seinen Funktionen ausführlicher als Evernote-Web und erlaubt eine Anpassung der Oberfläche und Funktionen auf die eigenen Vorlieben.

\section{Import-Ordner}

Mit dem Desktop-Client ist es zudem möglich, Import-Ordner aus dem Filesystem zu definieren, deren Inhalt (nur Bild und PDF beim kostenlosen Account) automatisch in ein definiertes Notizbuch importiert werden. So können Sie feste Routinen für die Übergabe von Daten an Evernote schaffen, die das manuelle Verschieben überflüssig machen.

\section{Gespeicherte Suchen}

Wenn Sie bestimmte Suchen in Evernote immer wieder durchführen, können Sie diese als feste Suchen speichern. Die gespeicherten Suchen werden dann auch in Evernote-Web angezeigt. Evernote bietet verschiedene Suchbefehle an, um auch komplexe Suchen zu ermöglichen.

Diese Anfrage sucht z. B. nach Notizen der vergangenen Woche, die Bilder enthalten und auf Ihrem iPhone erstellt wurden: 
Die Übersicht aller Suchfunktionen und -befehle finden Sie unter: [dev.evernote.com/ documentation/cloud/chapters/search_grammar.php]

\section{Textteile verschlüsseln}

Wenn Sie sensible Daten in Evernote speichern, können Sie diese auch verschlüsseln. Das geht bisher nur mit Textteilen, nicht aber mit ganzen Notizen. Dazu werden Sie von Evernote gebeten, eine Passphrase einzugeben. Diese gilt es sich gut zu merken, denn wenn Sie sie vergessen, sind die verschlüsselten Textstellen nicht wieder herstellbar.

\section{App}

Die App bietet in erster Linie die Möglichkeit, neue Inhalte schnell aufzunehmen und die vorhandenen Inhalte zu durchsuchen. Ich kann aber auch erweiterte Funktionen wie das Teilen von Inhalten über die App steuern.

\section{Inhalte teilen}

Es ist möglich, Inhalte oder Notizbücher mit anderen zu teilen, indem ich bestimmte Inhalte für andere freigebe.

Grundsätzlich kann man

- Notizen über soziale Netzwerke wie Facebook und Twitter teilen

- Notizen per E-Mail teilen

- Notizen über einen Link teilen

- Notizbücher mit anderen Evernote-Nutzern teilen (beim kostenlosen Account kann man nur Leserechte vergeben, keine Bearbeitungsrechte)

- Öffentliche Notizbücher erstellen

\section{Evernote-Web}

In der Weboberfläche verwenden Sie zum Freigeben von Inhalten den Button „Freigabe“ oder bei den Notizbüchern das Kontextmenü, das Sie über einen Rechtsklick auf ein Notizbuch öffnen können.

\section{Desktop}

In der Desktopoberfläche gibt es ebenfalls einen Button für die Freigabe, und auch hier kann für die Freigabe von Notizbüchern das Kontextmenü genutzt werden.

\section{App}

Auch die verschiedenen Apps von Evernote erlauben das Freigeben von Notizbüchern oder einzelnen Notizen über die jeweilige Oberfläche. In der Regel verbergen sich die Freigabeoptionen hinter diesem Symbol:

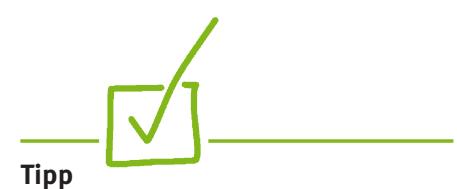

Evernote in der Schule: Blogbeitrag von Felix Schaumburg zum Einsatz von Evernote in der Schule:

[evernote-de.tumblr.com/ post/19178865591/wie-lehrer-felixschaumburg-mit-evernote-seinen] Evernote for School: [www. evernote.com/about/schools]

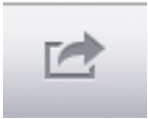




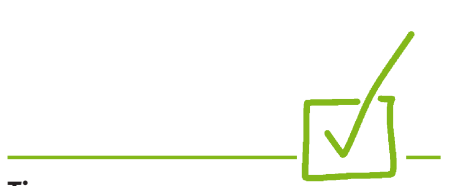

Tipp

Evernote Clearly [www.evernote. com/clearly] ist ein Add-on für die Browser Firefox und Chrome, mit dessen Hilfe man Webseiten auf den eigentlichen Text reduzieren kann: Alle Werbung, Banner, Zusatzinformationen werden ausgeblendet. Und mit einem Klick können Sie diesen Text, der auch Links enthält, dann als einen Eintrag in Evernote hinzufügen.

\section{Die Evernote Galerie}

Evernote bietet um ihr Kernprodukt noch eine Reihe ergänzender Produkte an. Teils für den Computer, teils als Apps. Diese verfügen über eine nahtlose Integration in Evernote und können einzelne Zusatz-Funktionen bieten.

Dazu gehören z. B. das Bildbearbeitungsprogramm Skitch (IOS, Adroid, MAC), Evernote People und Food (iPhone), beides visuelle Gedächnisstützen, die mit Inhalten in Evernote verknüpft werden können, sowie die Browsererweiterung Clearly (Firefox, Chrome), die es erlaubt, Inhalte, die beim Lesen einer Webseite stören, auszublenden.

Ein weiteres Evernote-Werkzeug ist Evernote Peek für das iPad. Mit Evernote-Peek können Sie aus Ihren Notizen Lernunterlagen erstellen und sich so Ihr eigenes, individuelles Lehrbuch schaffen, z. B. um die Matheaufgaben für den Sohn aufzupeppen, es im Unterricht mit Schülern für die eigenen Lehrinhalte zu nutzen oder Ihr Wissen in einem bestimmten Fachgebiet spielerisch aufzufrischen.

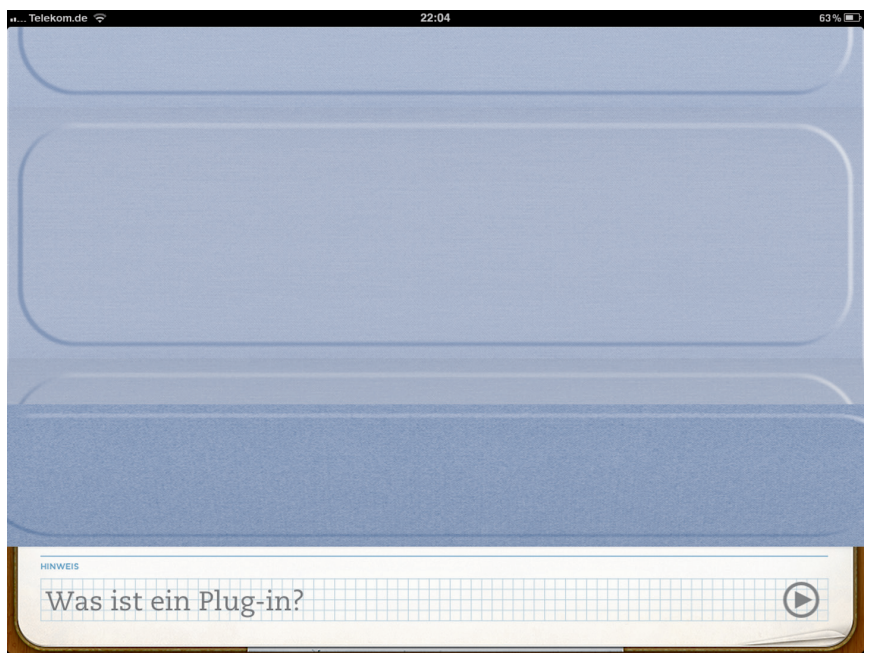

Um ein eigenes Lehrbuch zu erstellen, legen Sie einfach ein neues Notizbuch an. Evernote-Peek verwendet den Notiztitel als Lernfrage und den Notizinhalt als Antwort. Beides sollte nach Möglichkeit kurz und knackig formuliert sein. Zum einen, weil es sich so leichter lernt, zum anderen, weil Peek nur eine begrenzte Textlänge anzeigt.

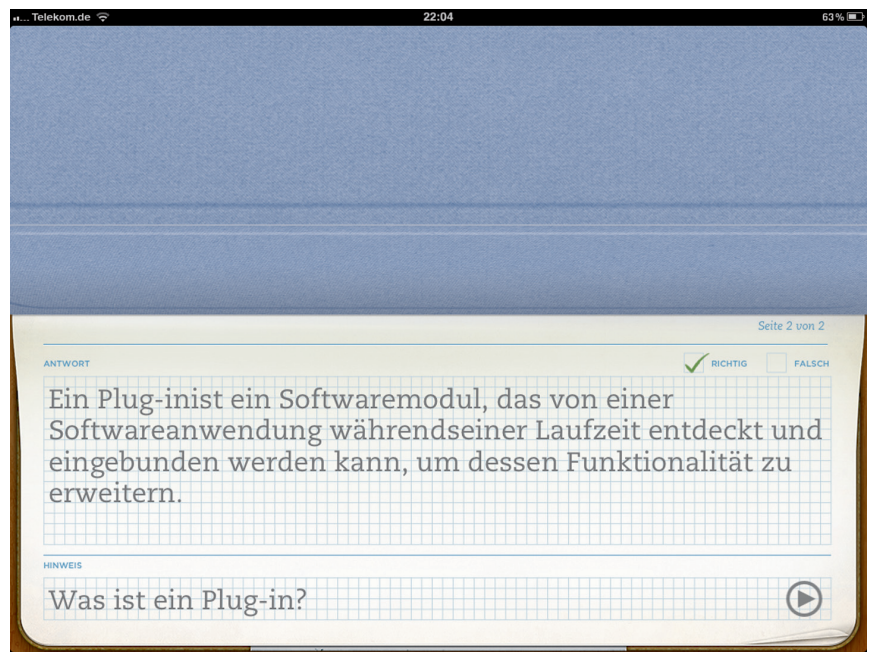

Und so sieht es dann aus. Viel Spaß beim Lernen! 


\section{Springpad}

Springpad [springpad.com] ist genauso wie Evernote ein „digitaler Zettelkasten“, hat aber eine stärkere Betonung auf ToDo-Listen und Sozialen Netzwerken. Springpad ist eine reine Webanwendung und bietet keinen zusätzlichen Desktop-Client an, wie Evernote es tut. Letztendlich ist es eine Geschmacksfrage, welches der beiden Tools, Springpad oder Evernote, einem eher zusagt.

Der Springpad-Account ist grundsätzlich kostenlos und es gibt keine Beschränkungen durch Datenvolumensgrenzen. Mit Hilfe des Chrome Add-on kann Springpad auch Offline genutzt werden.

\section{Neue Inhalte aufnehmen}

Browser

\section{Web-Clipper Add-on}

Zum Aufnehmen neuer Inhalte und zum Durchsuchen bereits vorhandener Inhalte im Schnellzugriff bietet Springpad das Web-Clipper Add-on für Firefox und Chrome an. Insgesamt bietet das Chrome Add-on einige Funktionen mehr (z. B. Offline-Modus, send to Phone), als das Firefox Add-on.

Um neue Inhalte aus dem Web aufzunehmen oder einfach eine Notiz oder eine Aufgabe einzugeben, klicken Sie auf das Browser Add-on. Es öffnet sich ein kleines Bedienfenster, über das Sie Zugriff auf Ihre Notizbücher und die Suche haben und über das Pluszeichen neue Inhalte eingeben können. Hier stehen folgende Optionen zur Verfügung:

- Ausschnitt aus der Webseite, auf der Sie sich gerade befinden bzw. ganze Seite

$$
\text { speichern }
$$

- Notiz hinzufügen

- Aufgabe hinzufügen

- Look it up (nur englische Quellen)

Beim Speichern der Inhalte haben Sie dann noch die Möglichkeit, den Speicherort (Notizbuch) auszuwählen, den Titel anzupassen und Tags hinzuzufügen.

Bei Webseiten wird ein Screenshot der Seite an die Notiz angehängt.

Nach dem Speichern gibt es die Möglichkeit, über einen Button den Webdienst aufzurufen, um die Notiz weiter zu bearbeiten.

Wenn Sie mit dem Chrome Add-on arbeiten, haben Sie zusätzlich die Möglichkeit, die Notiz an Ihr Android-Smartphone zu senden. Dazu müssen Sie die Spingpad App auf Ihrem Android-Smartphone installiert und mit dem Account verbunden haben.

Alternativ zum Add-on bietet Springpad ein Bookmarklet zum Sammeln von Inhalten an.

\section{"Look it up"-Funktion}

Die „Look it up“-Funktion von Springpad fügt zu gesuchten Inhalten wie Filmen, Produkten oder Orten aus bestimmten Quellen des Internets automatisch zusätzliche Informationen hinzu. Sucht man (in den USA) z. B. nach einem Kinofilm, ergänzt Springpad automatisch eine Liste mit Kinos und Spielzeiten.

Leider gibt es bei Springpad nicht die Möglichkeit, die Sprachoberfläche auf Deutsch anzupassen. Schön wäre in diesem Zusammenhang auch die Möglichkeit, für die Funktion „Look it up“ die Region Deutschland auswählen und somit deutschsprachige Quellen durchsuchen zu können. 
Da dies leider nicht geht, stehen für „Look it up“ ausschließlich englischsprachige Quellen zu Verfügung, was diese Funktion im deutschsprachigen Raum weitestgehend uninteressant macht.

App

Springpad bietet seine App für Android und IOS an. Die Apps für Smartphone und Tablets sind unterschiedlich gestaltet und bieten unterschiedliche Optionen. Hierbei gibt es noch kleine Unterschiedeim Funktionsumfang zwischen IOS und Android.

Als Beispiel verwenden wir hier ein iPhone und ein iPad, um die Funktionen zu beschreiben.

Das IOS App bietet Zugriff auf alle Notizbücher und gespeicherten Inhalte. Zudem ist es möglich, neue Inhalte aufzunehmen und zu bearbeiten. Für das Aufnehmen neuer Inhalte stehen folgende Optionen zur Verfügung:

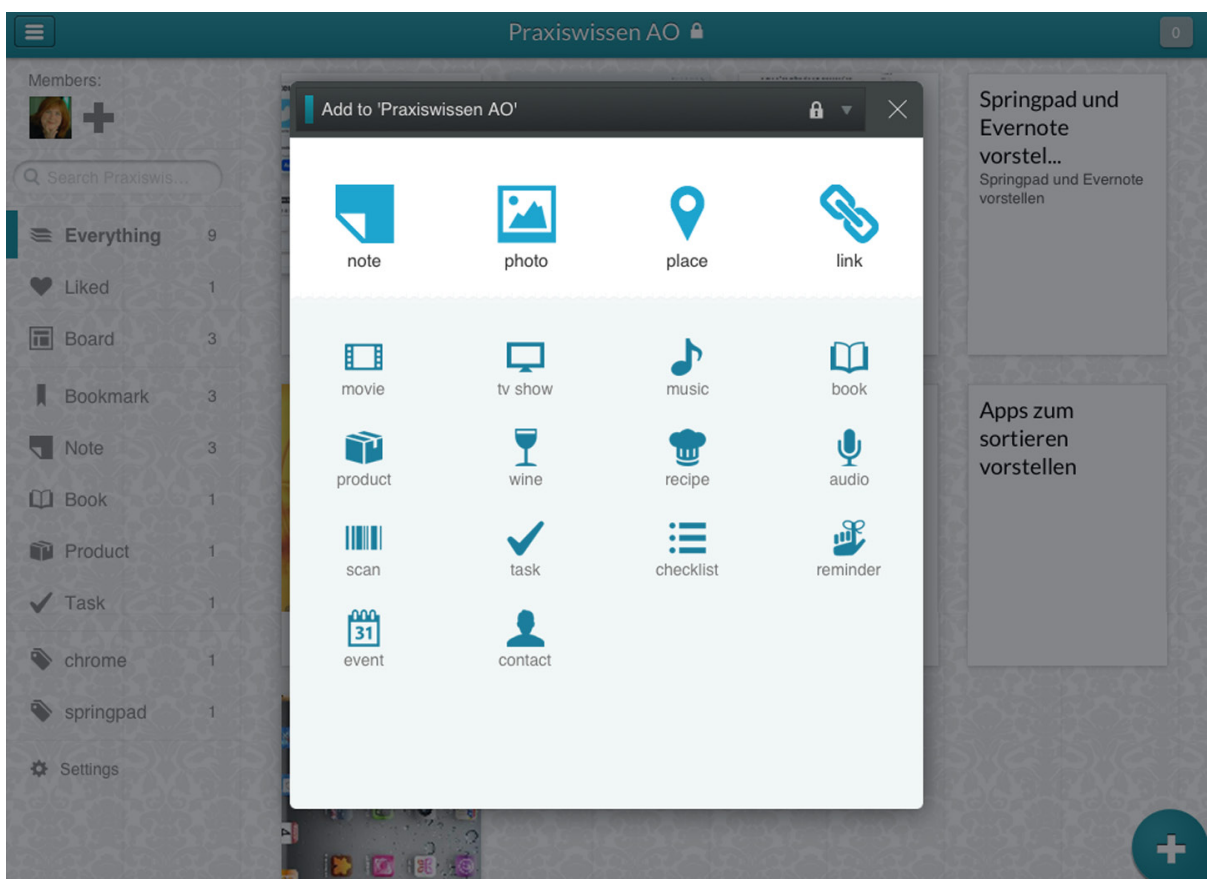

Add by type enthält verschiedene vorgefertigte Templates für das Aufnehmen von Informationen: 


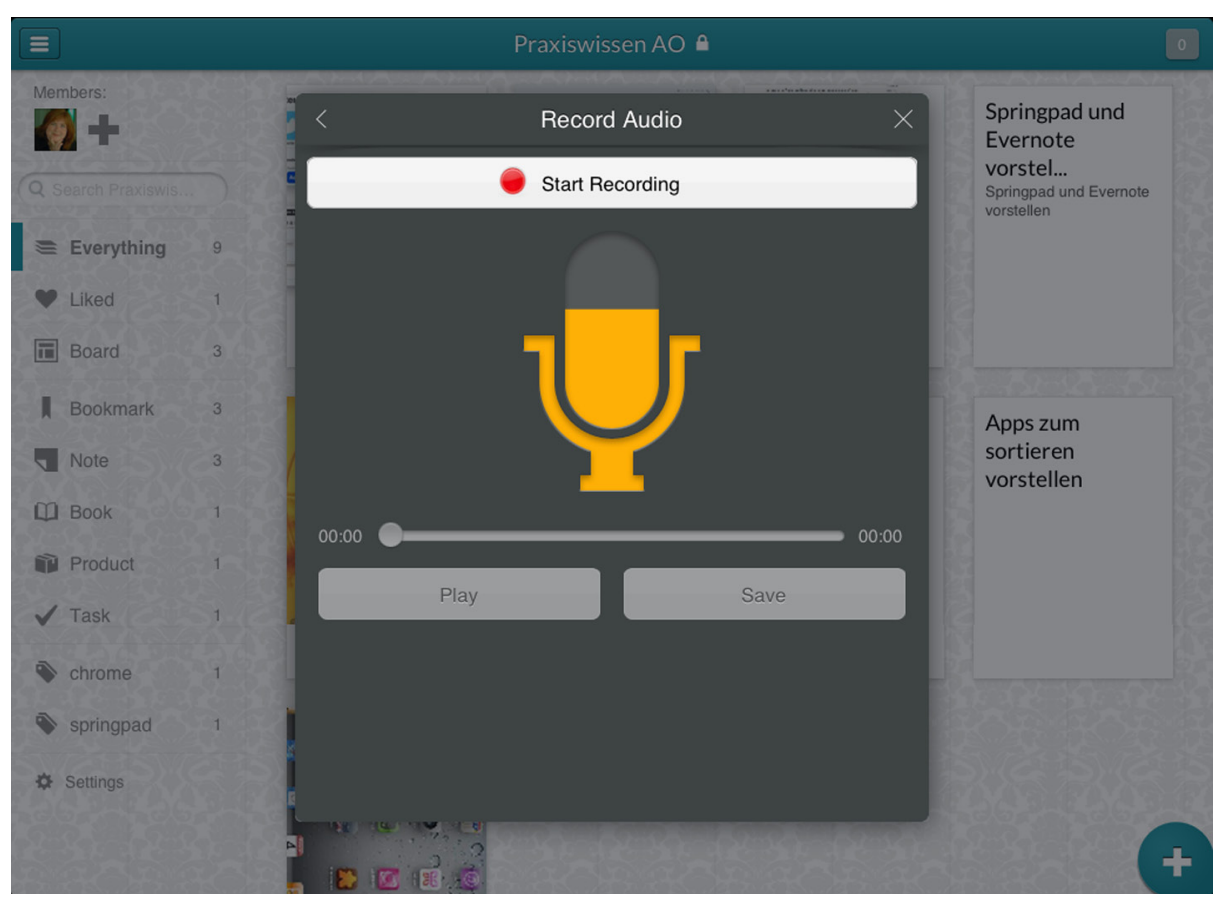

Zusätzlich zu den Funktionen, die wir schon aus dem Browser Add-on kennen, ist es hier auch möglich, eine Audionotiz abzuspeichern, sich Bücher mit Hilfe des Barcodescanners zu notieren oder eine Umgebungssuche durchzuführen, um so Informationen mit Ortsbezug zu finden und zu notieren.

Besonders die Übernahme von Informationen (Bücher, Produkte etc.) über den Barcode ist eine sehr gelungene Funktion, die auch das Auslesen von Informationen aus QR-Codes erlaubt. Der Barcode-Scanner beherrscht UPC (6, 12 digits), EAN (8, 13 digits) und QR-Codes.

\section{Springpad-Clipper für IOS}

Um auch über den Browser Ihres iPhones oder iPads Inhalte schnell in Springpad übernehmen zu können, ist es möglich, den Springpad-Clipper in den mobilen Safari-Browser zu integrieren. Die genaue Anleitung für die Installation von SpringpadClipper finden Sie in der App unter den Einstellungen.

\section{Inhalte verwalten und wiederfinden}

Browser

Die Inhalte sind bei Springpad in Notizbüchern organisiert. Dabei unterscheidet Springpad in der Ansicht zwischen den eigenen Notizbüchern, Notizbüchern, denen man "folgt" und einer Auswahl von Notizbüchern, die man „entdecken“ kann, um ihnen dann ggf. zu folgen. 


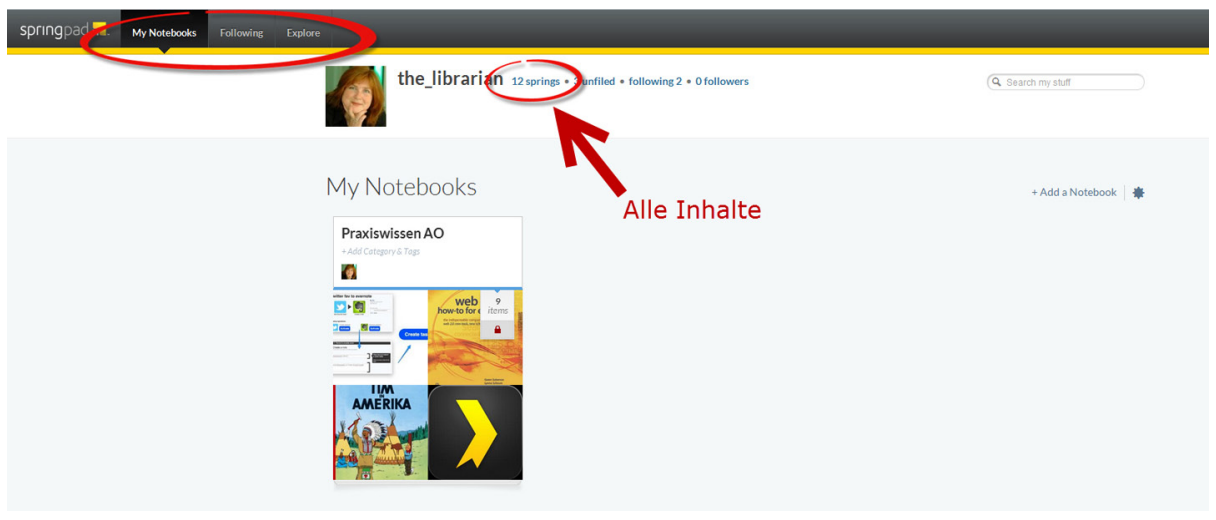

Von der Startseite aus kann man nun wählen, ob man Nachrichten aus seinen sozialen Netzwerken lesen, ein bestimmtes Notizbuch öffnen oder hinzufügen oder sich alle Inhalte in einer Sammlung anzeigen lassen möchte.

Wählt man die Anzeige aller Inhalte, kann man sie mit Hilfe fester Kategorien nach Informationstyp (wie z. B. Buch, Produkt, Notiz, Lesezeichen oder Aufgabe) filtern. Zudem steht eine Suchfunktion zur Verfügung.

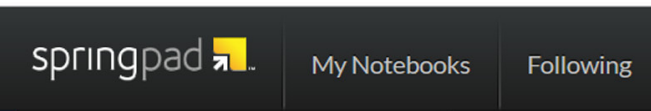

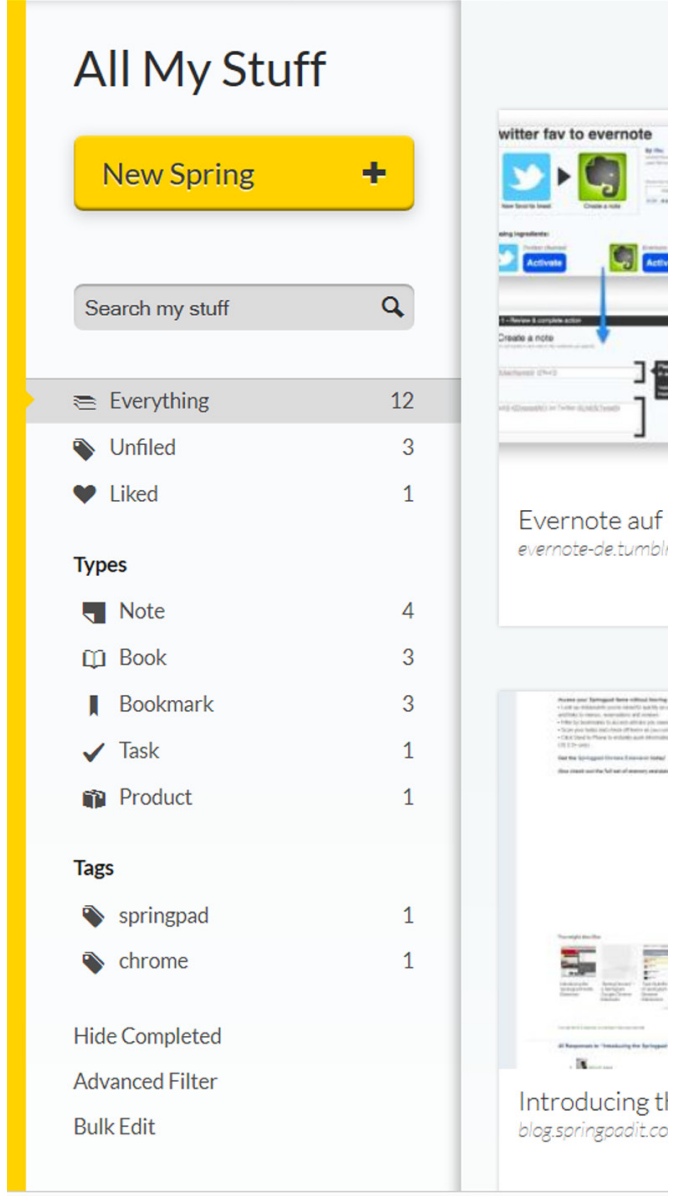


Wählt man eines seiner Notizbücher aus, steht einem neben den bereits erwähnten Filtern auch die Funktion „Board“ zur Verfügung.

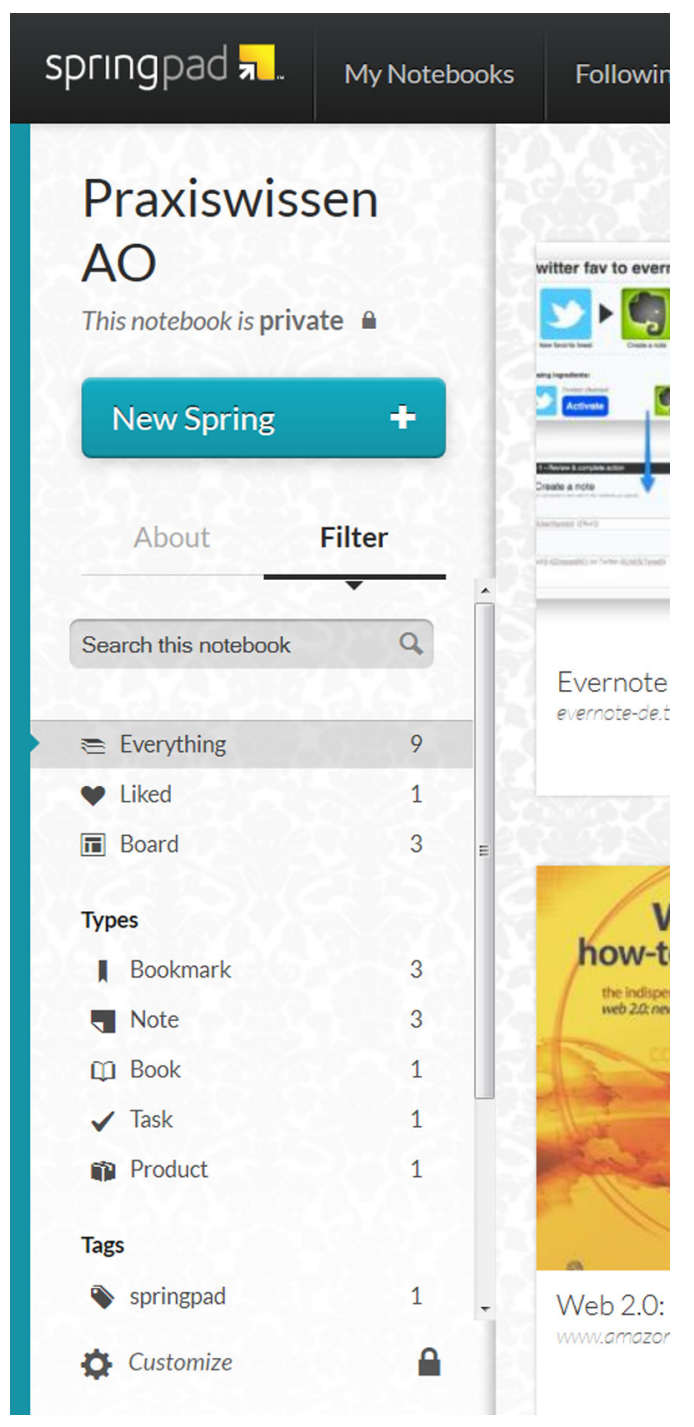

Über die Funktion Board kann man sich die Inhalte eines Notizbuches wie einzelne Bilder und Zettel auf einem virtuellen Tisch liegend anzeigen lassen und diese frei auf der Oberfläche bewegen und zueinander gruppieren.

Sie können auf dem Board Label platzieren, Heftnotizen schreiben, Landkarten einfügen und Inhalte aus Ihrem Notizbuch ablegen und gruppieren. Um Inhalte auf das Board zu legen, klicken Sie oben rechts auf „Add Items“. Nun öffnet sich ein Auswahlfenster, mit dessen Hilfe Sie Inhalte aus Ihrem Notizbuch auf dem Board ablegen können.

\section{Alerts}

Die Alert-Funktion erlaubt es, sich an bestimmte Ereignisse (News, Reminders, Deals) erinnern zu lassen, z. B. bei Terminen oder Aufgaben. Zusätzlich wird eine Meldung generiert, wenn ein Buch oder Produkt, welches Sie sich gemerkt haben, im Preis gesenkt wurde. Dabei bezieht sich der Dienst in erster Linie auf Amazon.com.

Um die Offline-Funktionalität von Springpad nutzen zu können, benötigen Sie den Browser Chrome und das dazugehörige Chrome Add-on für Springpad. 
App

Die iPhone-App bietet die Möglichkeit, sich die Inhalte in Listen anzeigen zu lassen, Inhalte zu suchen und zu bearbeiten. Die iPad-App bietet zusätzlich die Funktion „Board“. Auch hier können die Inhalte durch Ziehen frei auf dem virtuellen Tisch bewegt und platziert werden.

Sowohl die iPhone- als auch die iPad-App bieten die Möglichkeit, die Inhalte nach Kategorien und Tags zu filtern. Auch die Filter Alerts, Flagged Stuff und Board (beim iPhone in Listenansicht) stehen zur Verfügung.

\section{Inhalte teilen}

Browser

Die Komponente des Sozialen Netzwerkes ist bei Springpad besonders ausgeprägt. Daher gibt es hier umfangreiche Funktionen zum Teilen von Inhalten über Springpad oder soziale Netzwerke. Sie können Ihr Notizbuch z. B. mit Personen teilen, die Sie zur Zusammenarbeit per E-Mail einladen.

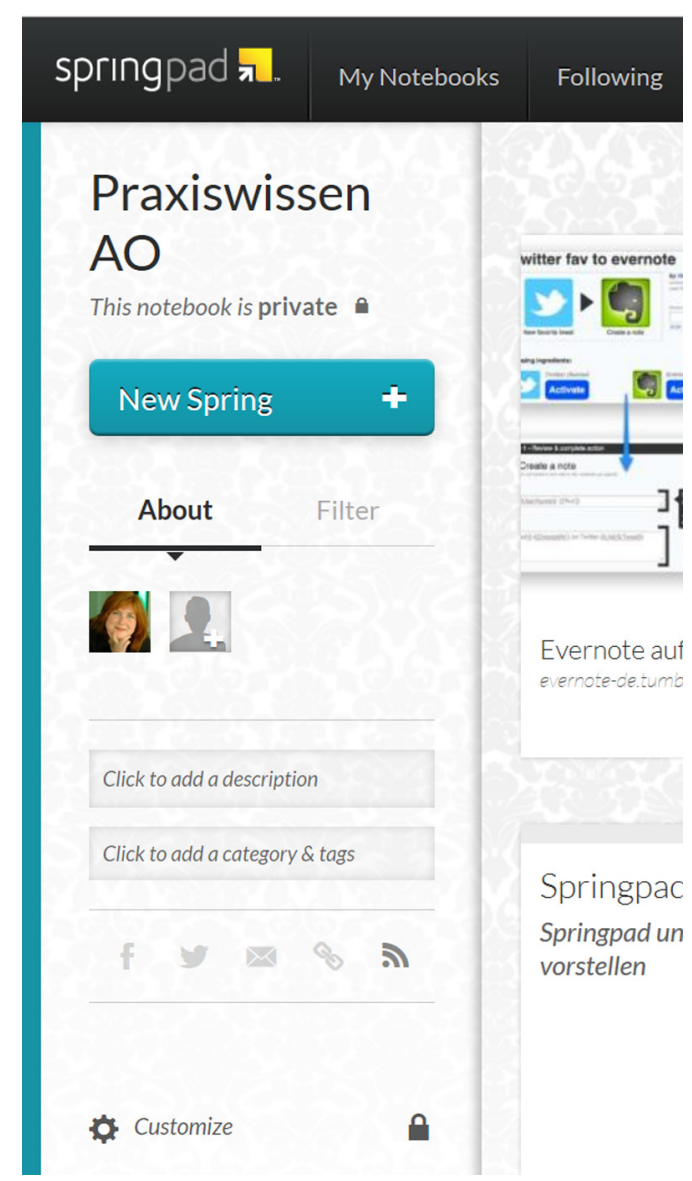

Um Ihr Notizbuch für ausgewählte Einzelpersonen via E-Mail freigeben zu können, müssen diese ebenfalls einen Springpad-Account haben.

Sie können Notizbücher aber auch öffentlich machen, dann können andere Springpad-Nutzer Ihrem Notizbuch „folgen“.

Es ist zusätzlich möglich, Ihren Springpad-Account mit Ihrem Twitter- oder Facebook-Account zu verbinden, um Neuigkeiten aus Ihren Notizbüchern über diese Webdienste $\mathrm{zu}$ teilen. 
Eine weitere Funktion von Springpad ist die Möglichkeit, „Öffentlichen Notizbüchern" anderer zu folgen. Diese können Sie sich in einer gesonderte Ansicht anzeigen lassen und den Neuerungen in diesen, meist thematisch ausgerichteten Notizbüchern, folgen.

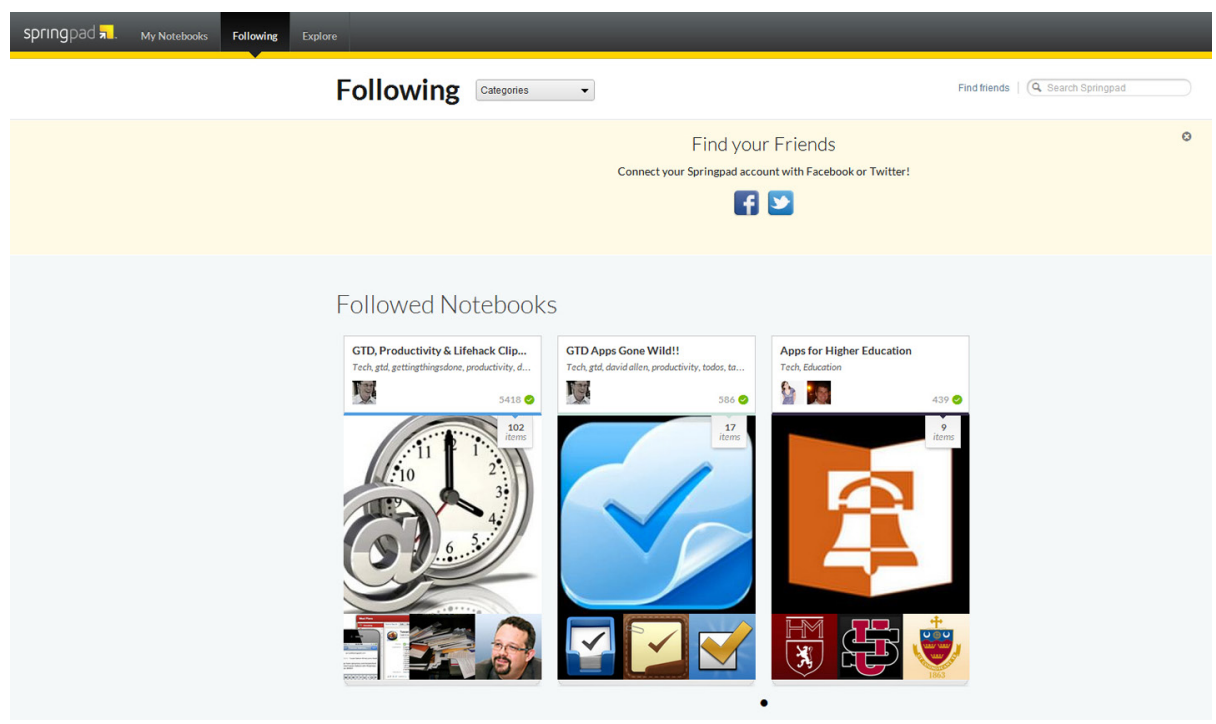

Followed Springs

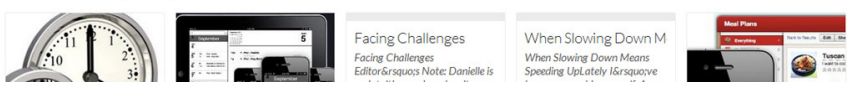

App

Auch über die App können Sie die Neuigkeiten aus anderen Notizbüchern verfolgen und Inhalte über Facebook und Twitter teilen.

Über die iPad-App ist es ebenfalls möglich, Notizbücher als „öffentlich“ zu markieren. Sie können jedoch keine Personen zu einem privaten Notizbuch zur Zusammenarbeit einladen, wie Sie es über die Webseite im Browser tun können.

\subsubsection{Notizbücher für den Desktop}

\section{Scribble Papers}

Das Auffinden von Werkzeugen zum Sammeln diverser Informationen, also digitaler Zettelkästen, ist oftmals erschwert durch die unterschiedliche Benennung und Eingruppierung in großen Softwareverzeichnissen.

Wenn Sie z. B. auf dem Softwareverzeichnis von heise.de [www.heise.de/download] nach Notizbüchern suchen, so finden Sie nicht alle passenden Werkzeuge, da sie verschiedenen Kategorien zugeordnet sind und oft nicht einmal das Wort „Notizbuch“ in Titel oder Beschreibung führen.

Ein „Klassiker“ im Bereich „Notizbücher“ ist beispielsweise Scribble Papers, das Sie entweder im heise.de-Softwareverzeichnis oder auf seiner Homepage, [www. scribblepapers.de.vu] finden können. Sie können in Scribble Papers Textstellen, Dateien, Bilder, Tabellen etc. ablegen und erschließen, ebenso Weblinks oder Dateien auf Ihrer Festplatte. Sie können das Notizbuch (oder den Zettelkasten, um ein anderes Bild zu verwenden) gut strukturieren und an Ihre Bedürfnisse anpassen. Eine

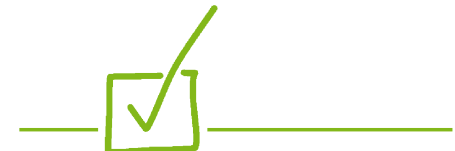

Tipp

Als Notizbuch würde sich auch ein Wiki eignen. Wikis müssen nicht unbedingt im Netz oder auf einem Server liegen. Beispielsweise gibt es ein Wiki, das in einer einzigen Datei untergebracht ist, TiddlyWiki [www.tiddlywiki.com]. Es ist sehr anpassungsfähig, und man kann es beispielsweise auf einem Stick als Notizbuch mit sich führen.

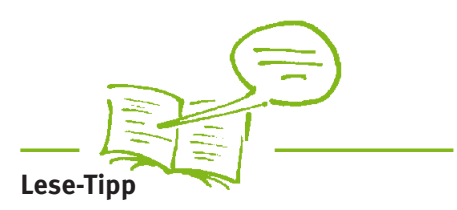

Wenn Sie einen elaborierten „Zettelkasten" für das wissenschaftliche Arbeiten suchen, der auch mit Literaturverwaltungsprogrammen Daten auszutauschen vermag, sollten Sie sich den „Zettelkasten nach Luhmann" ansehen: [zettelkasten. danielluedecke.de] 


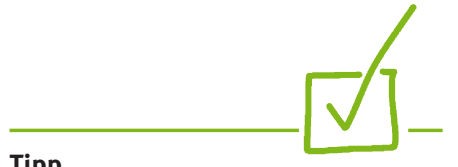

Tipp

Wenn es nur um schnelle Notizen geht, die bald wieder abgearbeitet werden sollen, dann sind Sticky Notes genau das Richtige: Post-its, die auf dem Bildschirm kleben, bis sie gelöscht werden. Ein Erinneremich wie die echten gelben Zettel! Windows 7 hat sie schon an Bord, Sticky Tool [www.heise.de/download/stickytool-1172661.html] erweitert die Funktionalitäten.

Diverse Programme wie Diigo oder Browser wie Opera bieten ebenfalls diese Möglichkeit, das Zettelchen bleibt aber im Fenster. Frei auf dem Desktop verschiebbar sind die

Sticky Notes [download.cnet.com/ Sticky-Notes/3000-2074 4-75560734.html] gute Suchfunktion ergänzt die strukturierte Ablage. Scribble Paper ist auch auf einem USB-Stick lauffähig.

\section{Quick Note}

Quick Note ist im Grunde ebenfalls ein Desktop-Notizbuch, verfolgt aber ein anderes Konzept als beispielsweise Scribble Papers. Es sitzt als Add-on im Browser (Firefox und Chrome) und es ermöglicht reine Textnotizen, auch Links sind möglich. So können Sie schnell eine Passage einer Webseite markieren und in eine Notiz stecken. Oder Sie können schnell Quick Note in einem Extrafenster öffnen und selbst eine Notiz erstellen.

Wenn Sie Quick Note und die Notizen auf mehreren Rechnern nutzen und somit synchronisieren möchten, so ist das in Zusammenarbeit mit dem sozialen Bookmarkdienst Diigo möglich. 